\title{
Spreading Factor Assignment Strategy for Coverage and Capacity Flexible Tradeoff
}

\author{
Luiz Q. R. da C. Filho, Alvaro A. M. de Medeiros, Jéssika C. da Silva, Vicente A. de Sousa Jr. and Níbia S. Bezerra
}

\begin{abstract}
LoRa is a physical layer technology with the ability to connect multiple devices in a wide area of coverage, with low power consumption and with interference robustness. The LoRaWAN specification introduces the protocol for communication between multiple devices and the gateway and defines an algorithm for spreading factor allocation. In this Letter, we investigate the efficiency of LoRa to send multiple uplink streams, analyzing different spreading factor allocation strategies to bring light to the coverage-capacity tradeoff. Additionally, we present a complete open-source simulation framework based on ns-3 simulator that can be used to propose, test and analyze the performance of new algorithms or heuristics that may outperform LoRaWAN ADR or any other baseline strategies defined here.
\end{abstract}

Index Terms-LoRaWAN, Spreading Factor, ns-3, IoT.

\section{INTRODUCTION}

A well-known Low-Power Wide Area Network (LPWAN) technology is the LoRaWAN [1], which is based on the physical layer technique LoRa (Long Range) in order to guarantee a robust signal over noise and interference, even in wider area deployments. LoRa technology, which is based on Chirp Spread-Spectrum (CSS), uses signals with different Spreading Factors $(S F \mathrm{~s})$ orthogonal to each other, so that it is possible to choose different factors for different devices at the same location, reducing interference between devices transmitting simultaneously. A higher $S F$ is allocated to End Devices (ED) located far from the Gateway (GW) enhancing robustness instead of throughput. A lower $S F$ can be allocated to EDs closer to GW, in order to increase throughput and reduce the frequency bandwidth occupancy (air time). Therefore, the $S F$ allocation plays a major role in the LoRaWAN network operation.

The LoRaWAN specification determines a policy for $S F$ assignment for the devices, namely Adaptive Data Rate (ADR), based on the ED's received power level: the higher the received power level is, the lower the $S F$ value is assigned.

Due to the growing interest in LoRaWAN, several studies [2]-[5] made an analytical performance evaluation, and the LoRaWAN perfomance is also analyzed via real network tests [6]-[9] and using ns-3 [10]-[14], OMNeT++ [15], [16] and LoRaWANSim [17] simulators.

In this Letter, we present a flexible strategy for $S F$ assignment that can be configured for different network

Luiz Filho and Alvaro Medeiros are with Federal University of Juiz de Fora (e-mails: \{luiz.quirino, alvaro\}@engenharia.ufjf.br.). Jéssika Silva e Vicente Sousa are with Federal University of Rio Grande do Norte, Brazil (e-mails: \{jessie,vicente.sousa\}@ufrn.edu.br). Níbia Bezerra is with Luleà University of Technology, Sweden (e-mails: nibia.souza.bezerra@ltu.se). This study was financed in part by the Coordenação de Aperfeiçoamento de Pessoal de Nível Superior - Brasil (CAPES) - Finance Code 001. The proof of concept simulations provided by this Letter was supported by High Performance Computing Center (NPAD/UFRN).

Digital Object Identifier: $10.14209 /$ jcis.2022.5 scenarios. The simplicity of such strategy allows a direct implementation on equipment with hardware constraints and an interesting analysis considering both viewpoints: capacity and coverage. We also compare our proposal with the ADR algorithm and other allocation policies that are focused only on either capacity or coverage. Our key contributions are the following:

- a simple and generic $S F$ allocation strategy that can be configured to increase capacity or coverage, and

- an open and available framework for both capacity and coverage performance evaluation based on ns-3 [18].

This Letter is organized as follows. Section II briefly introduces LoRa and LoRaWAN, and presents the $S F$ allocation methods evaluated in this Letter. Section III describes the simulation scenarios, and Section IV analyzes the obtained results. The final considerations are presented in Section V.

\section{Spreading Factor Assignment Methods}

LoRa is a technology patented by Semtech Corporation [19]. The term LoRaWAN refers to the architecture and specification of MAC layer, developed by the LoRa Alliance. Although the LoRa technology is proprietary, the LoRaWAN protocol is open source [20], and specifies parameters such as frame format, device classes and security protocols [21].

The LoRa modulation, which is based on a variation of CSS modulation, as so as the operation in sub $1 \mathrm{GHz}$ bands, are responsible for the high range and resistance to interference and noise. Its bit rate $R_{b}$ is defined as

$$
R_{b}=\frac{S F}{T_{S}}=S F \frac{B W}{2^{S F}}
$$

where $S F$ is the Spreading Factor, which determines the number of bits in the symbol and can vary from 7 to 12 . In addition, $S F$ determines the duration of a symbol $T_{s}$, which is the time required to scan the entire band of the signal $B W$. Although a higher $S F$ signal has a lower data rate, it is more tolerant to interference or noise due to easier detection at the receiver. Thus, the choice of $S F$ represents a tradeoff between transmission range and data rate.

LoRaWAN standards also defines the Adaptive Data Rate (ADR) algorithm that manages the power and data rate of the ED, by assigning its $S F$ [21]. After a configurable number of packages, the ED sends a request for an acknowledgement (ACK). If it does not receive the ACK, it tries to reestablish the connection by increasing the power and the $S F$, while reducing the data rate.

Thus, as each Spreading Factor requires a minimum received power to operate properly, the ADR algorithm consider the receiver sensitivity for each $S F$ before its assignment. Table I 
presents the receiver sensitivity and the bit rate for each $S F$ [19]. The larger the quantity of EDs operating with the same $S F$, the higher is the probability of interference, and consequently, the packet error rate.

TABLE I

SENSITIVITY AND BIT RATE FOR EACH $S F$.

\begin{tabular}{|c|c|c|}
\hline SF & Sensitivity $(\mathbf{d B m})$ & Bit rate $(\mathbf{k b} / \mathbf{s})$ \\
\hline $\mathbf{7}$ & -123 & 5.468 \\
\hline $\mathbf{8}$ & -126 & 3.125 \\
\hline $\mathbf{9}$ & -129 & 1.757 \\
\hline $\mathbf{1 0}$ & -132 & 0.976 \\
\hline $\mathbf{1 1}$ & -134.5 & 0.537 \\
\hline $\mathbf{1 2}$ & -137 & 0.293 \\
\hline
\end{tabular}

In this Letter, we analyze simple $S F$ assignment algorithms that can be applied to EDs with modest processing capabilities and may not generate extra traffic on the network. For this, we define the assignment vector $\mathbf{a}=\left\{a_{7}, a_{8}, \ldots, a_{12}\right\}$, where $a_{7}$ denotes the fraction of total EDs that are allocated to SF 7, $a_{8}$ to SF 8 , and so forth. Thus, we have that $\sum_{i=7}^{12} a_{i}=1$. Nine different methods of Spreading Factor allocation are evaluated

- Fixed at the lowest $S F$ (I) - All EDs use SF 7 (highest data rate), i.e. $a_{7}=1$ and $a_{i}=0 \forall i \neq 7$.

- Fixed at the highest $S F$ (II) - All EDs use SF 12 (lowest data rate), i.e. $a_{12}=1$ and $a_{i}=0 \forall i \neq 12$.

- Equally divided (III) - The $N$ EDs are ranked by received power and equally divide into six groups in a way that the the first group is allocated to SF 7, the second to SF 8 and so forth $\left(a_{i}=\lfloor N / 6\rfloor\right)$.

- Arbitrarily divided - The $N$ EDs are ranked and divided like method (III), but the quantity of EDs per group is an arbitrary fraction given by the assignment vector $\mathbf{a}$. We define two assignment vectors:

- Capacity enhancement (IV) - The algorithm follows $\mathbf{a}=\{0.6,0.2,0.05,0.05,0.05,0.05\}$.

- Coverage enhancement (V) - The algorithm follows $\mathbf{a}=\{0.05,0.05,0.05,0.05,0.2,0.6\}$.

- Sensitivity based (VI) - Each ED has the lowest $S F$ possible, so that the power received from that ED is greater than its sensitivity. This policy corresponds to ADR defined in LoRaWAN standards and the assignment vector depends on the deployment factors, such as position of EDs, wireless channel, and interference.

- Sensitivity based arbitrarily divided - This method follows the Arbitrarily divided one, but respecting the sensitivity rule defined in method (VI). This strategy affords $S F$ allocations that may differ from LoRaWAN ADR algorithm, since some EDs may be assigned to higher or lower SFs, improving coverage or capacity, respectively. We evaluate this strategy using two assignment vectors:

- Capacity enhancement (VII) - The algorithm follows $\mathbf{a}=\{0.6,0.2,0.05,0.05,0.05,0.05\}$, respecting the sensitivity rule defined by ADR.

- Coverage enhancement (VIII) - The algorithm follows $\mathbf{a}=\{0.05,0.05,0.05,0.05,0.2,0.6\}$ respecting the sensitivity rule defined by ADR.

- Randomly assigned (IX) - Each ED chooses a SF randomly from all six SFs with the same probability.

\section{Simulation Scenarios}

We use the version 3.29 of the ns-3 [22] for our evaluation, and the module developed by D. Magrin in [23], with a single GW multi-EDs deployment. There is no native LoRaWAN module for ns-3 and different modules can be used. Please refer to [10] for a more detailed description about the module we used and others proposed for ns-3.

In this way, a GW is placed surrounded by several EDs that send a packet in a random time within an interval of 10 minutes. Table II presents the parameters of simulations based on smart city scenario [12]. The log-distance path loss propagation model [24] was adopted so that we could focus on the capacity-coverage trade-off without randomness imposed by more complex channel models. However, since such channel models are defined in ns-3, this work can be extended to analyze the channel effects on the $S F$ allocation.

The LoRaWAN module on ns-3 adopted in this work is not fully implemented, and some MAC layer signaling is missing. As the $S F$ update is ideal, the results presented are considered for a steady-state network. There is no intra- $S F$ orthogonality. Collisions inter- $S F$ are handled in a quasi-orthogonal way, i.e. they can occur for high received power difference between simultaneous signals. Since the LinkADRReq command [21] is not implemented of this module, the configuration of transmission power and $S F$ is considered to be ideal.

TABLE II SiMULATION PARAMETERS.

\begin{tabular}{|c|c|}
\hline Parameter & Value \\
\hline Number of EDs & $1000,2000,3000,4000,5000,6000(\mathrm{~A})$ \\
& $1000,3000,6000(\mathrm{~B})$ \\
\hline Packet size & 23 Bytes \\
\hline Radius of circle & $3000,6000,10000 \mathrm{~m} \mathrm{(A)}$ \\
& $2000,3000,4000,6000,8000,10000 \mathrm{~m}(\mathrm{~B})$ \\
\hline Traffic direction & only uplink \\
\hline Bandwidth & $125 \mathrm{KHz}$ \\
\hline Path loss values & $d_{0}=1 \mathrm{~mL}\left(d_{0}\right)=7.7 ; n=3.7$ \\
\hline Transmission power & $14 \mathrm{dBm}$ \\
\hline Number of simulation runs & 10 \\
\hline
\end{tabular}

We define two sets of simulations, each one composed of three campaigns, to analyze the methods defined in Section II:

(A) Capacity analysis - Simulation campaigns are carried out with EDs uniformly distributed in a circle of radius of 3,6 and $10 \mathrm{~km}$, and the number of EDs increases as presented in Table II.

(B) Coverage analysis - Simulation campaigns are carried out with the number of EDs fixed at 1000, 3000 and 6000, and the radius of the circular area where they are uniformly distributed increases as shown in Table II.

\section{RESUlts}

Figures 1 and 2 show the percentage of correctly received packets and the throughput of all EDs, respectively, in function of the number of EDs for the $S F$ allocation methods presented in Section II. The 95\% confidence interval is represented by the shadow around the curves. We notice on Fig. 1(a) and 2(a) that for a smaller radius $(3000 \mathrm{~m})$, the coverage-based methods (II, V and VIII) present the worst performance, since many EDs are allocated with higher $S F$ s, increasing the probability of a collision (higher packet transmission time). Methods (III) 
and (IX) performs similarly, as many EDs do not have high $S F$. Methods (I) and (VI) perform better, since $S F 7$ provides the highest rates and the distance between EDs and the GW is low enough to prevent packet loss. In the LoRaWAN ADR method (VI), most EDs are configured with $S F$ 7, as the distance to GW is not high enough. The few EDs that operate below the sensitivity threshold are allocated to higher $S F$ s, so that they can also transmit. Methods (IV) and (VII) outperform LoRaWAN ADR and present the best performance since they allocate the EDs along all $S F$ s with fewer collisions occurring in $S F 7$.

As the radius increases (Figures 1(b), 1(c), 2(b), and 2(c)), we notice that the coverage-based algorithms improve their performance, since most of the EDs are allocated to high $S F \mathrm{~s}$, guarantying signal coverage and protection to interference. However, it can be observed from Figures 1(c) and 2(c) that strategies (I), (III) and (IV) increase the throughput and maintain their PDR level in the $10-\mathrm{km}$ scenario. Although strategies (I) and (IV) focus on $S F 7$ to enhance capacity, strategy (III) equally allocates EDs for all $S F$ s. This indicates that inter- $S F$ interference might play a major role in the capacity-coverage trade-off.

An interesting result is that methods (III) and (IX) outperform all methods including the LoRaWAN ADR method (VI), as both radius and number of EDs increase. This indicates that capacity and coverage can not be analyzed separately. As the number of EDs in a wider area increases, interference plays a major role. EDs with the same $S F$ tend to transmit with the same power, which means that a collision may be harmful for both packets. However, when EDs with different $S F$ s transmits simultaneously, the difference of transmission power and air time may result in a successful reception for at least one of them. As method (IX) outperforms method (III), we verify that finding the optimum allocation vector is not as simple as dividing $S F \mathrm{~s}$ equally. Furthermore, as the random allocation outperforms the LoRaWAN ADR method, improvements on the latter should consider other factors besides ED sensitivity. In fact, our simulation framework available in [18] can leverage the investigation of algorithms to define vector $\mathbf{a}$, and consequently, allocation methods in LoRaWAN systems.

Fig. 3 presents the results of the second set of simulations (B), where the circular area around the GW increases. It can be noticed that, for lower values of the circular area radius, the strategies follow the same tendency of simulation campaign (A), with strategy (II) presenting the worst performance. The LoRaWAN ADR method (VI) performs well for lower radius distances, as so as capacity-oriented methods such as (IV) and (VII). Furthermore, Fig. 3 show that the LoRaWAN ADR presents the best performance only for lower coverage radius. As the radius increases, the coverage-oriented algorithms (V) and (VIII) tend to maintain their performance, specially in scenarios with higher number of nodes. However, they do not outperform methods (III) and (IX) for the 6000-ED scenario, as also observed in simulation campaign (A).

\section{Final Remarks}

The Internet of Things concept has created a demand for wireless network technologies capable of supporting a large number of EDs with low communication rates and high energy efficiency. The LoRa technology and the LoRaWAN systems have been widely adopted for such purpose.

In this Letter, we evaluated several ways of Spreading Factor allocation strategies in order to analyze the capacity-coverage tradeoff of LoRaWAN networks. In comparison to LoRaWAN ADR algorithm, we evaluated simple methods of allocating $S F$ s that do not demand greater processing load, energy consumption or relevant alterations on LoRaWAN standard.
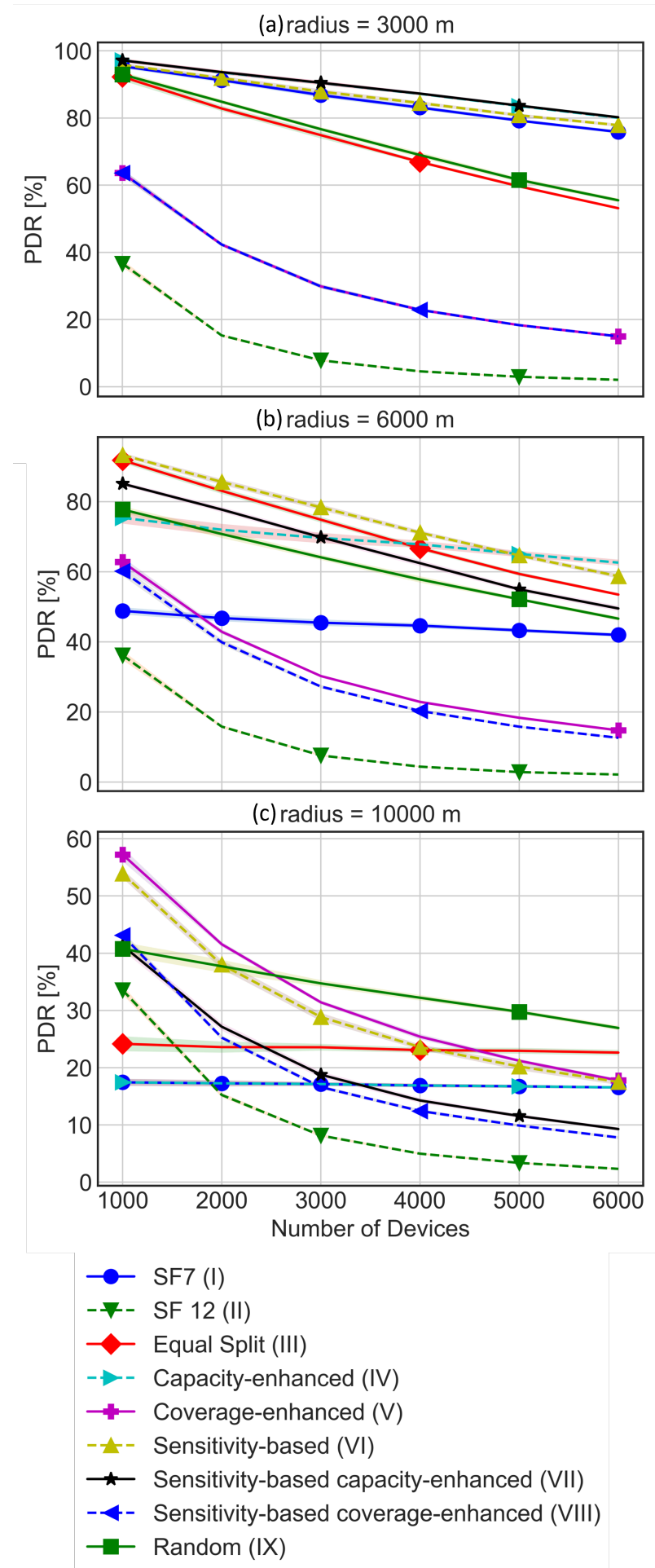

Fig. 1. Percentage of received packets in function of the number of EDs for different $S F$ allocation policies for EDs uniformly distributed

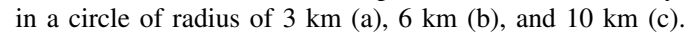



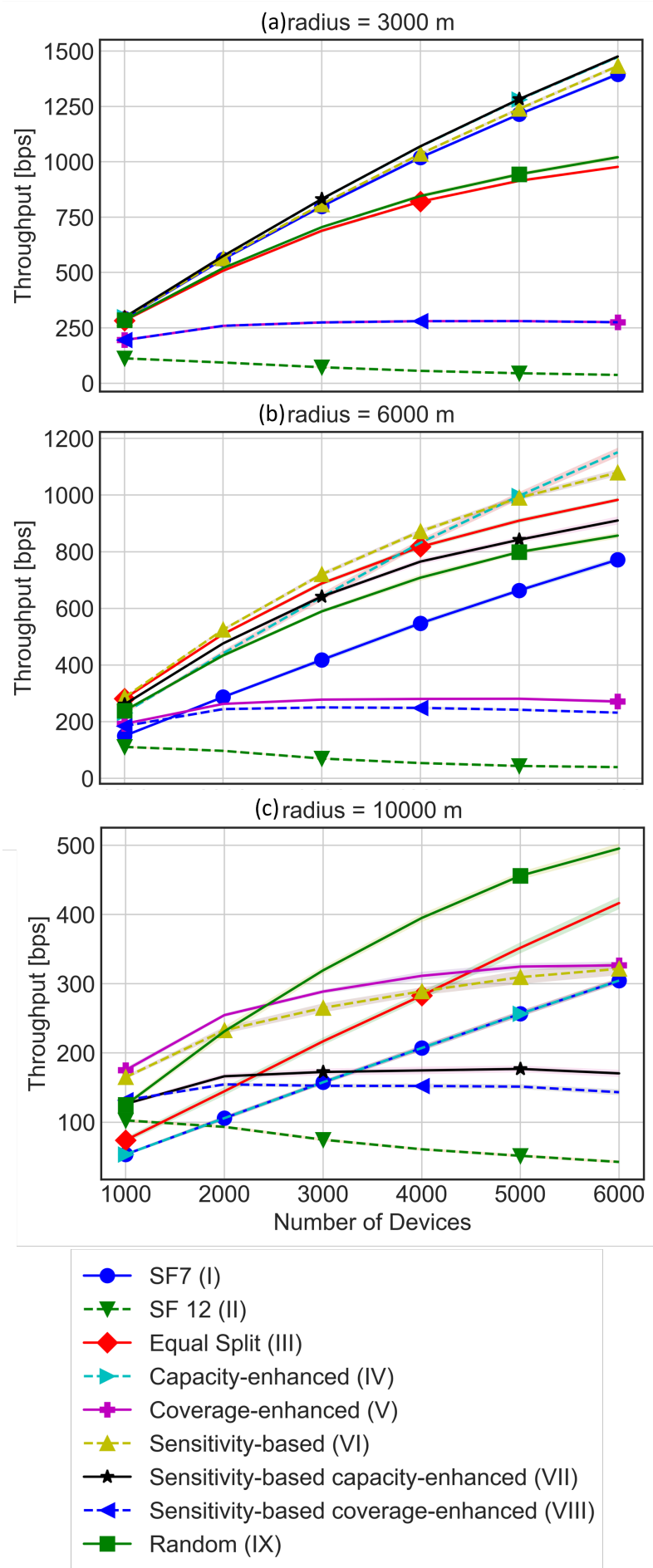

Fig. 2. Throughput of the network in function of the number of EDs for different $S F$ allocation policies for EDs uniformly distributed in a circle of radius of $3 \mathrm{~km}$ (a), $6 \mathrm{~km}$ (b), and $10 \mathrm{~km}$ (c).

The utilization of a proper $S F$ allocation method is crucial to the network operation. For instance, in a network with 6000 EDs, depending on the $S F$ allocation method, the number of received packets increases from $15 \%$ to $80 \%$, and the network throughput from $40 \mathrm{bps}$ to $180 \mathrm{bps}$. Such difference defines the type of service provided by the network.

For wider area networks, as the number of EDs increases, we noticed that neither coverage nor capacity enhancement algorithms are the optimum solutions for the $S F$ allocation problem, as the interference between $S F$ s becomes a relevant factor. For this reason, the development of optimum $S F$
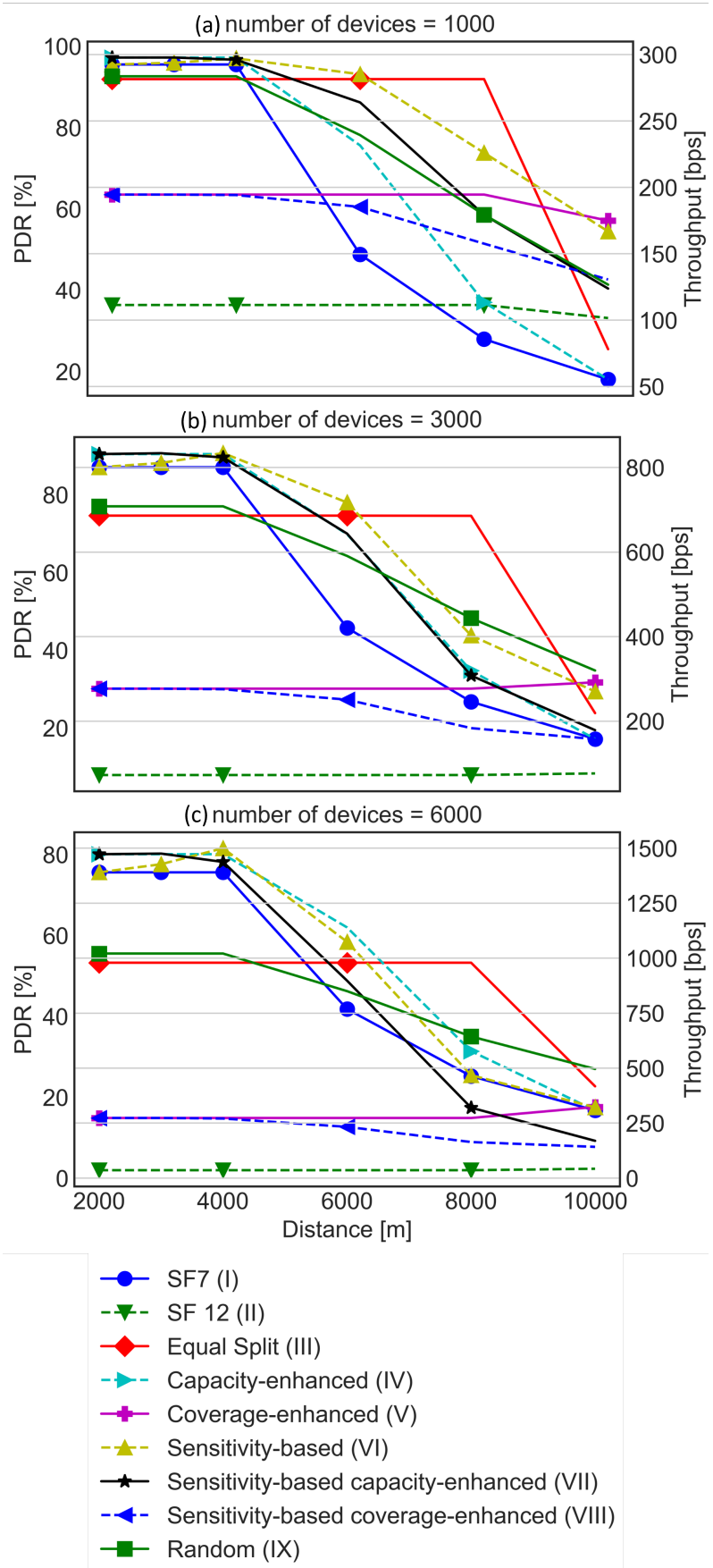

Fig. 3. PDR and Throughput in function of the circular area radius size for different $S F$ allocation policies for a number of EDs fixed at 1000 (a), 3000 (b), and 6000 (c).

allocation algorithms is challenging task.

The framework provided in this Letter [18] can be used to design and test $S F$ allocation algorithms that can be easily implemented in practical LoRaWAN deployments. A simple Spreading Factor strategy based on the assignment vector a is proposed where one can easily evaluate the performance comparing to LoRaWAN ADR and other baseline strategies presented in this Letter. Factors such as EDs' position, mobility and traffic patterns and quality of service constraints can also be used to find the proper allocation vector a for each application scenario. Our future works include the investigation of machine learning solutions based on such factors in a dynamic scenario. 


\section{REFERENCES}

[1] W. Ayoub, A. E. Samhat, F. Nouvel, M. Mroue, and J. Prévotet, "Internet of Mobile Things: Overview of LoRaWAN, DASH7, and NB-IoT in LPWANs Standards and Supported Mobility," IEEE Communications Surveys Tutorials, vol. 21, no. 2, pp. 1561-1581, Secondquarter 2019, DOI: https://doi.org/10.1109/COMST.2018.2877382.

[2] A. Mahmood, E. Sisinni, L. Guntupalli, R. Rondón, S. A. Hassan, and M. Gidlund, "Scalability Analysis of a LoRa Network Under Imperfect Orthogonality," IEEE Transactions on Industrial Informatics, vol. 15, no. 3, pp. 1425-1436, March 2019, DOI: https://doi.org/10.1109/TII.2018.2864681.

[3] R. M. Sandoval, A. Garcia-Sanchez, J. Garcia-Haro, and T. M. Chen, "Optimal Policy Derivation for Transmission Duty-Cycle Constrained LPWAN,” IEEE Internet of Things Journal, vol. 5, no. 4, pp. 3114-3125, Aug 2018, DOI: https://doi.org/10.1109/JIOT.2018.2833289.

[4] H. Mroue, A. Nasser, B. Parrein, S. Hamrioui, E. Mona-Cruz, and G. Rouyer, "Analytical and Simulation study for LoRa Modulation," in 201825 th International Conference on Telecommunications (ICT), June 2018, pp. 655-659, DOI: https://doi.org/10.1109/ICT.2018.8464879.

[5] S. Ruben M., G.-S. Antonio-Javier, and G.-H. Joan, "Performance optimization of LoRa nodes for the future smart city/industry," in EURASIP Journal on Wireless Communications and Networking, 2019, DOI: https://doi.org/10.1186/s13638-019-1522-1.

[6] M. Chiani and A. Elzanaty, "On the LoRa Modulation for IoT: Waveform Properties and Spectral Analysis," IEEE Internet of Things Journal, vol. 6, no. 5, pp. 8463-8470, Oct 2019, DOI: https://doi.org/10.1109/JIOT.2019.2919151.

[7] T.Polonelli, D.Brunelli, and A. Marzocchi, "Slotted ALOHA on LoRaWAN-Design, Analysis, and Deployment," Sensors (Basel), 2019, DOI: https://doi.org/10.3390/s19040838.

[8] A. Carlsson, I. Kuzminykh, R. Franksson, and A. Liljegren, Measuring a LoRa Network: Performance, Possibilities and Limitations, 09 2018, pp. 116-128, DOI: https://doi.org/10.1007/978-3-030-01168-0_11.

[9] N. Souza Bezerra, C. Åhlund, S. Saguna, and V. Sousa, "Temperature Impact in LoRaWAN-A Case Study in Northern Sweden," Sensors, vol. 19, p. 4414, 10 2019, DOI: https://doi.org/10.3390/s19204414.

[10] J. Silva, D. Flor, V. A. d. Junior, N. Bezerra, and A. Medeiros, "A Survey of LoRaWAN Simulation Tools in ns-3," Journal of Communication and Information Systems, vol. 36, no. 1, pp. 17-30, Feb. 2021, DOI: https://doi.org/10.14209/jcis.2021.2.

[11] M. Capuzzo, D. Magrin, and A. Zanella, "Confirmed traffic in LoRaWAN: Pitfalls and countermeasures," in 2018 17th Annual Mediterranean Ad Hoc Networking Workshop (Med-Hoc-Net), June 2018, pp. 1-7, DOI: https://doi.org/10.23919/MedHocNet.2018.8407095.

[12] D. Magrin, M. Centenaro, and L. Vangelista, "Performance evaluation of LoRa networks in a smart city scenario," in 2017 IEEE International Conference on Communications (ICC), May 2017, pp. 1-7, DOI: https://doi.org/10.1109/ICC.2017.7996384.

[13] D. Magrin, M. Capuzzo, and A. Zanella, "A Thorough Study of LoRaWAN Performance Under Different Parameter Settings," IEEE Internet of Things Journal, vol. 7, no. 1, pp. 116-127, Jan 2020, DOI: https://doi.org/10.1109/JIOT.2019.2946487.

[14] A. D. Prajanti, B. Wahyuaji, F. B. Rukmana, R. Harwahyu, and R. F. Sari, "Performance Analysis of LoRa WAnTechnology for Optimum Deployment of Jakarta Smart City," in 2018 2nd International Conference on Informatics and Computational Sciences (ICICoS), Oct 2018, pp. 1-6, DOI: https://doi.org/10.1109/ICICOS.2018.8621803.

[15] F. Cuomo, M. Campo, A. Caponi, G. Bianchi, G. Rossini, and P. Pisani, "EXPLoRa: Extending the performance of LoRa by suitable spreading factor allocations," in 2017 IEEE 13th International Conference on Wireless and Mobile Computing, Networking and Communications (WiMob), Oct 2017, pp. 1-8, DOI: https://doi.org/10.1109/WiMOB.2017.8115779.

[16] M. Slabicki, G. Premsankar, and M. Di Francesco, "Adaptive configuration of lora networks for dense IoT deployments," in NOMS 2018 - 2018 IEEE/IFIP Network Operations and Management Symposium, 2018, pp. 1-9, DOI: https://doi.org/10.1109/NOMS.2018.8406255.

[17] S. Li, U. Raza, and A. Khan, "How Agile is the Adaptive Data Rate Mechanism of LoRaWAN?" in 2018 IEEE Global Communications Conference (GLOBECOM), Dec 2018, pp. 206-212, DOI: https://doi.org/10.1109/GLOCOM.2018.8647469.

[18] GppCom, "GppCom Github (LoRaWAN Repository)." [Online]. Available: \url\{https://github.com/vicentesousa/ns-3-lora-gppcom\}

[19] Semtech Corporation. (2015) AN1200.22 LoRa ${ }^{\mathrm{TM}}$ Modulation Basics. [Online]. Available: www.semtech.com/uploads/documents/an1200.22. pdf

[20] A. Lavric and V. Popa, "Internet of Things and LoRaTM Low-Power Wide-Area Networks: A survey," in 2017 International Symposium on Signals, Circuits and Systems (ISSCS), July 2017, DOI: https://doi.org/10.1109/ISSCS.2017.8034915.

[21] LoRa Alliance Technical Committee, LoRaWAN ${ }^{\mathrm{TM}} 1.1$ Specification, LoRa Alliance Std., 2017.

[22] NS-3, "Network simulator-3." [Online]. Available: https://www.nsnam. org/.

[23] D. Magrin, "Network level performances of a lora system." [Online]. Available: http://tesi.cab.unipd.it/53740/1/dissertation.pdf.

[24] T. S. Rappaport, Wireless Communications: Principles and Practice. Prentice Hall, 2002. 